\title{
EFFECTS OF INTER-SET STRETCHING ON ACUTE HORMONAL AND METABOLIC RESPONSE: A PILOT STUDY
}

original paper

( ) University School of Physical Education in Wroclaw

DOI: https://doi.org/10.5114/hm.2019.79218

\section{DOUGLAS POPP MARIN ${ }^{1,2}$, CHRISTIANO BERTOLDO URTADO ${ }^{3}$, CAMILA GUAZELI MARQUES ${ }^{1}$, ALLAN IGOR SILVA SERAFIM ${ }^{1,4}$, LUIS FELIPE TUBAGI POLITO ${ }^{1,4}$, FERNANDO NORONHA DE ALMEIDA ${ }^{5}$, JONATO PRESTES ${ }^{5}$, ROSEMARI OTTON ${ }^{2}$}

${ }^{1}$ Graduation Program in Physical Education, Methodist University of São Paulo, São Bernardo do Campo, Brazil

${ }^{2}$ Interdisciplinary Post-Graduate Program in Health Science, Cruzeiro do Sul University, São Paulo, Brazil

${ }^{3}$ Graduation Program in Physical Education, Federal University of Maranhão, São Luís, Brazil

${ }^{4}$ Post-Graduate Program in Physical Education, São Judas Tadeu University, São Paulo, Brazil

${ }^{5}$ Graduation Program in Physical Education, Catholic University of Brasilia, Brasilia, Brazil

\section{ABSTRACT}

Purpose. The present study aimed to compare the acute hormonal and metabolic response following a resistance training (RT) session with and without inter-set stretching.

Methods. Overall, 13 men with minimum 1 year of experience with RT were randomly allocated to 2 groups: traditional RT group (TRT; $n=6$; age: $22.5 \pm 4.7$ years; body weight: $83.1 \mathrm{~kg} \pm 5.7 \mathrm{~kg}$; height: $179.8 \pm 6.9 \mathrm{~cm}$; $\mathrm{BMI}: 25.73 \pm 1.17 \mathrm{~kg} / \mathrm{m}^{2}$ ) and stretching RT group (SRT; $n=7$; age: $23.4 \pm 4.3$ years; body weight: $82.3 \pm 11.7$ kg; height: $177.7 \pm 11.0 \mathrm{~cm}$; BMI: $26.02 \pm$ $2.5 \mathrm{~kg} / \mathrm{m}^{2}$ ). The RT protocol consisted of 6 bench press sets to failure at $80 \%$ of 8 repetitions maximum, with a $1-\mathrm{minute}$ rest interval between sets. During the rest interval, the TRT group remained resting, while the SRT group was submitted to shoulder anterior chain muscles stretching.

Results. The results revealed a higher increase in lactate (32\%) and total leukocytes (17\%) immediately following exercise in the SRT group as compared with the TRT group $(p<0.05)$. An increase in total testosterone and cortisol was observed in both groups, without differences between them $(p>0.05)$. There was a progressive decrease in the total number of repetitions completed along sets, without differences between groups.

Conclusions. The use of agonist stretching between sets increases the acute metabolic response during an RT session, which could be useful to intensify RT in trained participants.

Key words: muscle stretching, strength exercise, resistance training method, metabolic response

\section{Introduction}

Regular resistance training (RT) promotes chronic adaptations related to muscle and power increase, and muscle hypertrophy [1]. There are different RT components; each of them has a specific configuration of the acute variables, including intensity, volume, rest interval between sets, and exercise selection and order [2]. The manipulation of rest interval between sets alters the acute hormonal [3], metabolic [4], and neuromuscular response to RT. Some practitioners use a short rest interval (30-60 seconds) between sets to potentiate muscle hypertrophy, based on exacerbated acute immediate hormonal response [3], which could, in part, change cellular signalling and promote neuromuscular adaptations. The acute elevation in hormonal response during and immediately following exercise is associated with higher metabolic stress,

Correspondence address: Douglas Popp Marin, Cruzeiro do Sul University, Rua Regente Feijó, 1295, 03342-000,

São Paulo - SP, Brazil, e-mail: douglas.marin@metodista.br

Received: July 23, 2018

Accepted for publication: October 18, 2018

Citation: Marin DP, Urtado CB, Marques CG, Serafim AIS, Polito LFT, de Almeida FN, Prestes J, Otton R. Effects of inter-set stretching on acute hormonal and metabolic response: a pilot study. Hum Mov. 2019;20(1):55-61; doi: https://doi.org/10.5114/ hm.2019.79218. 
evidenced by lactate, inorganic phosphate, and hydrogen ion accumulation $[1,2]$. In theory, the acute increase in hormonal response would favour the interaction with receptors, inducing anabolic intracellular signalling [5].

Furthermore, the inter-set stretching of the agonist muscle has been applied to optimize the metabolic and hormonal responses to RT. The use of inter-set muscle stretching increases the time under tension and muscle blood flow restriction, which may raise neuromuscular, hormonal, and metabolic response [6]. The stretching of the agonist muscle between sets promotes some neuromuscular alterations, reduces the muscle blood flow, and increases the ischemic response during an RT session [7]. The higher accumulation of metabolites induced by inter-set stretching may elevate the blood concentration of anabolic hormones, such as growth hormone, insulin-like growth factor-1, and total testosterone [6].

Inter-set stretching has been shown to affect the acute performance of neuromuscular response [8, 9]. Do Rosário Souza et al. [10] investigated the effects of static stretching between sets during bench press and squat exercises. The authors found that inter-set stretching reduced the total number of repetitions completed and the performance of muscle strength.

Only one study evaluated the effects of stretching between sets on hormonal response. Souza et al. [7] found no alterations in resting cortisol or growth hormone following 8 weeks of RT with stretching between sets. Although their study was designed to investigate the chronic adaptations to this method, no research evaluated the acute hormonal response.

Thus, the present study aimed to compare the metabolic and hormonal response following acute RT with and without stretching between sets. The initial hypothesis is that inter-set stretching results in a more pronounced hormonal and metabolic stress response as compared with RT without stretching between sets.

\section{Material and methods}

\section{Participants}

The total of 13 male participants with more than 12 months of experience with RT were selected for the study. The subjects were healthy, did not present any contraindications to complete the tests and exercises proposed, did not use medications or supplements that could potentially interfere with the results. The division of the participants into 2 different groups followed the randomization and counterbalance criteria.
The groups were: a traditional RT group (TRT; age: $22.5 \pm 4.7$ years; body weight: $83.1 \mathrm{~kg} \pm 5.7 \mathrm{~kg}$; height: $179.8 \pm 6.9 \mathrm{~cm}$; BMI: $25.73 \pm 1.17 \mathrm{~kg} / \mathrm{m}^{2}$ ) and a stretching RT group (SRT; age: $23.4 \pm 4.3$ years; body weight: $82.3 \pm 11.7 \mathrm{~kg}$; height: $177.7 \pm 11.0 \mathrm{~cm}$; BMI: $26.02 \pm 2.5 \mathrm{~kg} / \mathrm{m}^{2}$ ). All participants were informed about the purposes, procedures, possible risks, and benefits of the study.

\section{Experimental procedures}

Data were collected during a 3-week period. First, the participants visited the laboratory to determine their anthropometric profile, to get familiarized with the RT equipment (Cybex Incorp, USA), and to complete the test of 8 repetitions maximum ( $8 \mathrm{RM})$. The following 2 weeks consisted of acute RT sessions. Blood samples were collected between 08:00 and 10:00 a.m., before and immediately after the RT sessions. The subjects were advised to remain fasting for 12 hours during the night before data collection and to avoid any exercise, medications, supplements, and alcoholic drinks for 48 hours before the tests. After the pre-exercise blood sample collection, the participants received maltodextrin $(0.7 \mathrm{~g} / \mathrm{kg})$ in $8 \%$ solution and initiated warm-up 30 minutes later.

\section{Strength testing}

The participants completed a bench press $8 \mathrm{RM}$ testing in accordance with the recommendations of Do Rosário Souza et al. [10]. The test was terminated at the moment when the subjects were unable to perform the complete movement or when the concentric failure occurred. The following strategies were adopted to reduce test variations: (1) standard instructions were given before the test so that the volunteer was aware of the entire routine to be performed during data collection; (2) the subject was provided with technical instruction on the exercise performance; (3) the evaluator was aware of the position adopted by the practitioner during the test, since slight variations in the positioning of the joints involved in the movement could trigger other muscles, leading to misinterpretation of the obtained scores; (4) the participants were verbally encouraged with the purpose to keep their motivation level high; (5) the weight plates used in the study were previously measured on a precision scale. The participants were allowed to rest for 3-5 minutes between trials with 3 attempts and the initial load determined in the familiarization session. 


\section{Acute protocols}

Both groups completed a cycle ergometer light warmup lasting 5 minutes and 8 repetitions of bench press at $50 \%$ of $8 \mathrm{RM}$. The participants were then submitted to 6 sets of bench press at $80 \%$ of 8 RM to concentric failure, determined by the inability to complete the concentric phase of the movement. The TRT group completed the protocol with a 1-minute rest interval between sets, while the SRT group applied passive static stretching of the shoulder internal-anterior chain muscles (chest and anterior deltoid) during the rest interval, as shown in Figure 1. The subjects remained lying on the bench with shoulders in horizontal abduction and elbows slightly flexed (Figure 1).

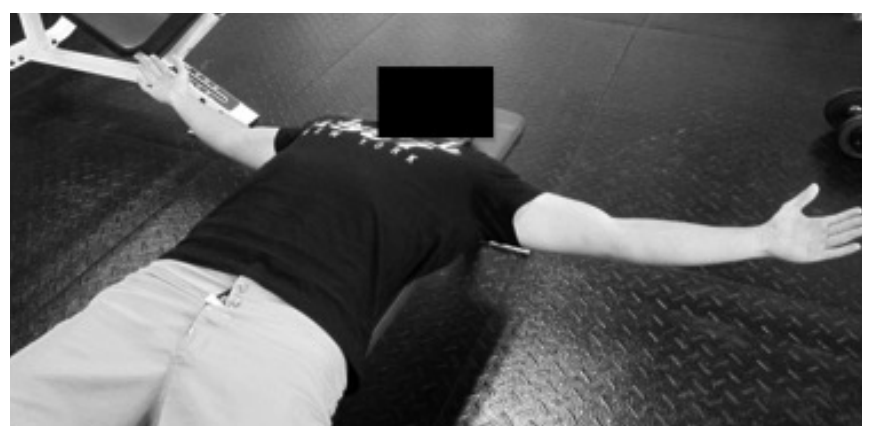

Figure 1. Stretching position

Fatigue index

The participants were advised to complete as many repetitions as possible in each set. The rate of decline in the number of repetitions per set was used to calculate the fatigue index as proposed by Sforzo and Touey [11]:

Fatigue index $=[$ (number of repetitions set $1-$ number of repetitions set 6$) /($ number of repetitions set 1$)] \times 100 \%$

\section{Biochemical analysis}

Blood samples of $3 \mathrm{ml}$ were collected from the antecubital vein into Vacutainer tubes (Becton Dickinson, São Paulo, Brazil). The samples were centrifuged at $800 \mathrm{~g}\left(4^{\circ} \mathrm{C}, 10\right.$ minutes). Plasma lactate was determined by the colorimetric method (Weiner Lab, São Paulo, Brazil). The lactate present in the sample is oxidized by the lactate dehydrogenase of the reagent in the presence of oxygen, producing pyruvate and hydrogen peroxide. The hydrogen peroxide suffers the action of peroxidase that produces the chromogenic compost absorbed at 540/550 nm. Cortisol and total testosterone concentrations were evaluated with the use of a chemiluminescence kit (Roche, São Paulo, Brazil) in accordance with the kit recommendations. Total leukocytes count was determined by impedance.

\section{Statistical analysis}

The data are expressed as mean \pm standard deviation $(S D)$. Shapiro-Wilk and Mauchly's tests were applied to check for normality and sphericity, respectively. Repeated measures ANOVA (group $\times$ time) was used, and alpha levels were set at 0.05. The Greenhouse-Geisser correction of degrees of freedom was utilized when sphericity was violated. Tukey's honest significant difference (HSD) post-hoc test was used when appropriate and the differences in the number of repetitions between sets were verified by the polynomial orthogonal contrast test. Eta partial squared was calculated for each ANOVA as a measure of effect size. The threshold values for small, moderate, and large effects were assumed to be $0.01,0.06$, and 0.14 , respectively. The analyses were performed with the SPSS software, version 22.0 (SPSS Inc., Chicago, USA).

\section{Ethical approval}

The research related to human use has been complied with all the relevant national regulations and institutional policies, has followed the tenets of the Declaration of Helsinki, and has been approved by the local Research Ethics Committee for Human Use (2.639.316/2018).

\section{Informed consent}

Informed consent has been obtained from all individuals included in this study.

\section{Results}

Repeated measures ANOVA revealed a significant difference in the bench press total number of repetitions $\left(\mathrm{F}(1.57,17.3)=26.290 ; p<0.001 ; \eta_{\mathrm{p}}^{2}=0.7\right)$, while there was no difference between groups $(\mathrm{F}(1,11)$ $\left.=0.73 ; p>0.05 ; \eta_{p}^{2}=0.007\right)$ and no interaction between groups and sets $(\mathrm{F}(1.57,17.3)=0.60 ; p>0.05$; $\left.\eta_{\mathrm{p}}^{2}=0.005\right)$. The contrast polynomial cubic test revealed a significant decrease in the number of repetitions across the sets $(\mathrm{F}(1,11)=12.19 ; p<0.01$ for both groups) (Figure 2).

Figures $3 \mathrm{~A}$ and $3 \mathrm{~B}$ present the total number of repetitions and the fatigue index, respectively. There was no difference in the total number of repetitions between protocols $(\mathrm{t}(11)=0.271 ; p>0.05)$. Similarly, 


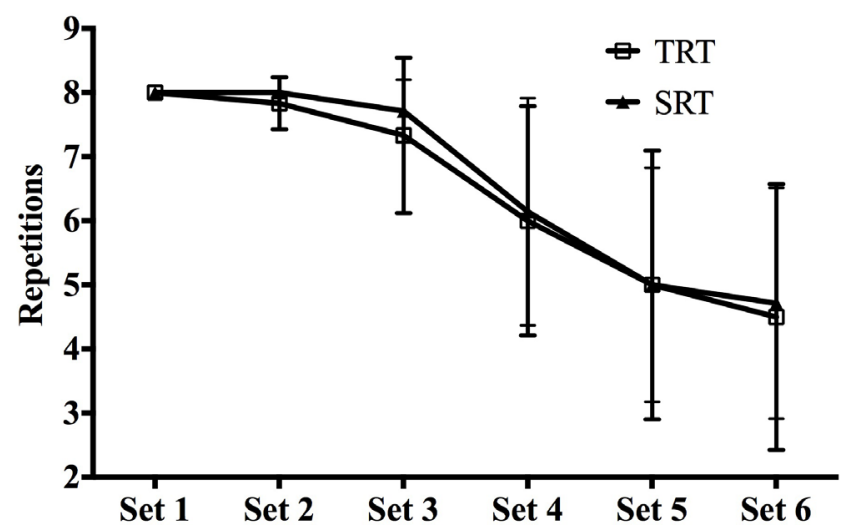

SRT - stretching resistance training, TRT - traditional resistance training

Figure 2. Number of repetitions completed per set of the bench press. Data as mean $\pm S D$

there was no difference in the fatigue index between groups $(\mathrm{t}(11)=-0.200 ; p>0.05$; SRT: $41.1 \pm 22.4 \mathrm{vs}$. TRT: $43.7 \pm 25.9)$.

Lactate levels were significantly higher after the training sessions $\left(\mathrm{F}(1,11)=194.56 ; p<0.01 ; \eta_{\mathrm{p}}^{2}=0.94\right)$. There was a difference between groups $(\mathrm{F}(1,11)=$ $\left.6.31 ; p<0.05 ; \eta_{\mathrm{p}}^{2}=0.36\right)$, but no interaction between time and groups $\left(\mathrm{F}(1,11)=6.108 ; p=0.08 ; \eta_{\mathrm{p}}^{2}=0.25\right)$. Tukey's HSD test revealed that lactate was higher in

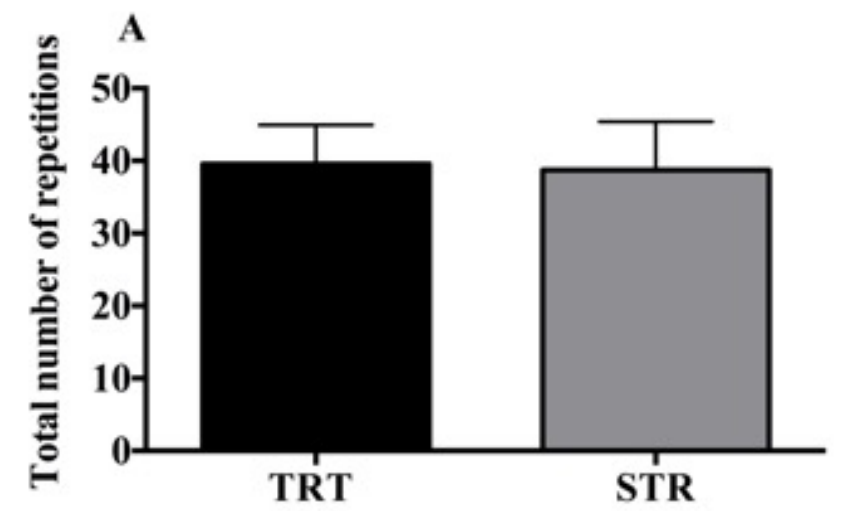

the SRT group (32\%) as compared with the TRT group $(p<0.05)$. Testosterone levels were increased after the protocols $\left(\mathrm{F}(1.11)=18.21 ; p<0.01 ; \eta_{\mathrm{p}}^{2}=0.62\right)$, while there was no difference between groups $(\mathrm{F}(1,11)$ $\left.=0.056 ; p>0.05 ; \eta_{\mathrm{p}}^{2}=0.005\right)$ and no time and group interaction $\left(\mathrm{F}(1,11)=0.022 ; p>0.05 ; \eta_{\mathrm{p}}^{2}=0.002\right)$. Cortisol levels were increased after the protocols $(\mathrm{F}(1,11)$ $\left.=25.33 ; p<0.01 ; \eta_{\mathrm{p}}^{2}=0.70\right)$, while there was no difference between groups $\left(\mathrm{F}(1,11)=1.29 ; p>0.05 ; \eta_{\mathrm{p}}^{2}=\right.$ $0.001)$ and no time and group interaction $(\mathrm{F}(1,11)=$ $0.08 ; p>0.05 ; \eta_{\mathrm{p}}^{2}=0.10$ ) (Table 1).

There was a significant increase in total leukocytes immediately following exercise $(\mathrm{F}(1,11)=5.27$; $\left.p<0.05 ; \eta_{\mathrm{p}}^{2}=0.32\right)$ and an interaction between time and group $\left(\mathrm{F}(1,11)=5.93 ; p<0.05 ; \eta_{\mathrm{p}}^{2}=0.35\right)$. Tukey's HSD post-hoc test revealed that total leukocytes increased only following the SRT session $(17 \% ; p<0.05)$ (Table 1).

\section{Discussion}

The present study aimed to compare the acute effect of RT with and without stretching between sets on the total number of repetitions, as well as metabolic, hormonal, and leukocytes response in trained men.

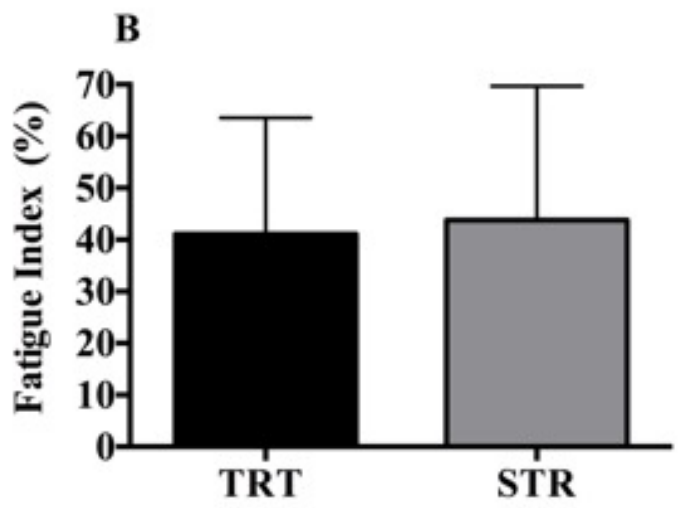

SRT - stretching resistance training, TRT - traditional resistance training

Figure 3. Total number of repetitions completed in the bench press (A) and fatigue index (B). Data as mean $\pm S D$

Table 1. Biochemical response pre- and post-exercise for each protocol

\begin{tabular}{lcccc} 
& \multicolumn{2}{c}{ SRT group } & \multicolumn{2}{c}{ TRT group } \\
\cline { 2 - 5 } & Pre-exercise & Post-exercise & Pre-exercise & Post-exercise \\
\hline Lactate $(\mathrm{mmol} / \mathrm{l})$ & $1.81 \pm 0.56$ & $9.87 \pm 2.13^{* \#}$ & $1.34 \pm 0.30$ & $7.46 \pm 1.56^{*}$ \\
Testosterone $(\mathrm{nmol} / \mathrm{l})$ & $447 \pm 129.5$ & $534.7 \pm 168.8^{*}$ & $461.8 \pm 92.3$ & $555.8 \pm 159.6^{*}$ \\
Cortisol $(\mathrm{nmol} / \mathrm{l})$ & $455.7 \pm 72.5$ & $561.8 \pm 71.2^{*}$ & $419.6 \pm 140$ & $587.6 \pm 150^{*}$ \\
Total leukocytes $\left(\right.$ per $\left.\mathrm{mm}^{3}\right)$ & $5428.5 \pm 1343.7$ & $6557.1 \pm 1626.7^{* \#}$ & $5616.6 \pm 1144$ & $5583.6 \pm 1342.2$ \\
\hline
\end{tabular}

Data as mean $\pm S D$.

SRT - stretching resistance training, TRT - traditional resistance training

* statistical significance at 0.05; compared with pre-exercise

* compared with the TRT group 
Both groups presented a decrease in the number of repetitions along the bench press sets, and an increase in cortisol and testosterone, with no differences between protocols. Lactate was 32\% higher following RT with inter-set stretching as compared with the protocol without stretching. Moreover, total leukocytes increased only after the protocol with inter-set stretching.

Several studies showed the acute negative effect of inter-set stretching on muscle strength $[9,12,13]$. The proposed mechanisms for the decrease in muscle strength include mechanical factors that alter the viscoelastic properties of the myotendinous unit and neural factors, such as a decrease in the activation of motor units. However, results from the total number of repetitions are controversial in the literature, and this is the first study to evaluate the acute metabolic stress induced by inter-set stretching during RT.

Nelson et al. [14] investigated physically active men and women submitted to a stretching protocol composed by 2 exercises to knee flexors with 4 sets of 30 seconds and 15 seconds of interval between exercises. The results revealed a $24 \%$ decrease in the total number of repetitions completed in the knee curl exercise. The performance was less affected with lower intensities ( $9 \%$ reduction at the intensity of $40 \%$ ) as compared with the session without stretching. In the present study, there was a decrease in the total number of repetitions among sets, without differences between protocols. Possible explanations include the different intensity ( $80 \%$ of $1 \mathrm{RM})$ and stretching used as compared with the study by Nelson et al. [14]. The results of the present study are in accordance with those obtained by Gomes et al. [15], who investigated the effects of static stretching on the maximum number of repetitions at $40 \%, 60 \%$, and $80 \%$ of $1 \mathrm{RM}$ and found no differences between the protocols with or without stretching at the intensity of $80 \%$. To note, the variations in the number of sets, exercises, strength performance evaluation, rest interval between sets, and time under stretching are widely variable between studies, which makes some comparisons difficult.

Fowles et al. [16] utilized 135 seconds of stretching observing a $25 \%$ decrease in the performance of maximum voluntary contractions. In the present study with 60 seconds of stretching, the decrease in the total number of repetitions along sets was similar between protocols. The lower time under stretching as compared with the study by Fowles et al. [16] may not be sufficient to decrease motor units activation and muscle electromyographic activity, while higher lactate and leukocytes were observed following the inter-set stretching protocol.
Moreover, in the present study, the participants were advised to remain in the stretched position, but not forced stretching, which may be considered a passive static procedure with a low overload on the muscle. As described by Funk et al. [17], this type of stretching may not impact on muscle performance, especially in trained participants. Figures $3 \mathrm{~A}$ and $3 \mathrm{~B}$ illustrate that there was no difference between protocols in the drop of total repetitions and fatigue index. Lopes et al. [18] found a higher decrease of $12 \%$ in the total number of repetitions with a stretching protocol, with no difference in the fatigue index. Ribeiro et al. [19] observed no difference between the impact of stretching before the bench press and no stretching on the total number of repetitions completed at $80 \%$ of $1 \mathrm{RM}$, or on the fatigue index. It seems that stretching before RT does not affect the fatigue index as reported by Ribeiro et al. [19] and Lopes et al. [18].

The results of the present study revealed an interesting issue. Although the stretching protocol showed no additional negative effect on repetitions performance, it resulted in higher metabolic stress (32\% more lactate) and higher total leukocytes count as compared with the TRT protocol. The increase in total leukocytes may be associated with the increase in cortisol [20], as this hormone stimulates immune cell migration from bone marrow to the bloodstream, and, later, to damaged tissues [21]. Evidence from an experimental study showed that blood flow decreased proportionally with muscle stretch [22]. Furthermore, Mayhew et al. [23] found that a 1-minute rest interval between sets resulted in higher total leukocytes as compared with that of 3 minutes. Kruse et al. [24] demonstrated that in humans retrograde muscle blood flow decreased following stretching. Otsuki et al. [25] observed a decrease in blood volume accompanied by a general decline in microvascular muscle oxygenation induced by stretching. McCully [26] also demonstrated that the combination of stretching and isometric contractions resulted in a dramatic decrease in muscle oxygenation.

Mohamad et al. [6] suggest that stretching between sets may induce hypertrophy to a greater extent than TRT. Although it is tempting to correlate the acute increase in testosterone with muscle hypertrophy, this mechanism is a matter of strong debate in the literature [27]. There is evidence linking lactate (increasing the phosphorylation of the p70S6K enzyme, a target of the mTORC1 anabolic pathway) increase with an elevation of circulating testosterone and cortisol [28]. Lactate has been proposed as an anabolic signalling molecule [28, 29]. 
The present study has some limitations, such as the limited number of participants in each group, as well as the lack of evaluation of the range of motion gains during stretching, direct measures of hypoxia, tissue damage, and patterns of muscle activation. The inclusion of more mechanisms, different types of stretching, and molecular pathways could help to understand the effects of inter-set stretching. To note, participants from the present study were trained and may have had limited responsiveness capacity as compared with untrained people, which reinforces the importance of the results.

In the literature, there are studies that evaluated the effect of stretching on performance, including a study by Walsh [30], who found an increase in the proprioception characteristics and a decrease in strength at the same time, especially with static stretching. On the other hand, despite the limitations enumerated above, this is the first study to evaluate the influence of inter-set stretching on metabolic and hormonal parameters.

\section{Conclusions}

The data obtained from the present study suggest that 60 -second stretching between sets during the bench press exercise does not interfere with total repetitions along sets, while it results in higher metabolic stress and total leukocytes count. Long-lasting studies should confirm that higher acute metabolic stress is accompanied by higher muscular adaptations.

\section{Acknowledgments}

The authors thank the laboratory of clinical analysis of the Methodist University of São Paulo and the Coordination of Improvement of Higher Educational Personnel (CAPES), the participants involved in the research, and the Cruzeiro do Sul University.

\section{Disclosure statement}

No author has any financial interest or received any financial benefit from this research.

\section{Conflict of interest}

The authors state no conflict of interest.

\section{References}

1. Schoenfeld BJ. Postexercise hypertrophic adaptations: a reexamination of the hormone hypothesis and its applicability to resistance training program design. J Strength Cond Res. 2013;27(6):1720-1730; doi: 10.1519/JSC.0b013e31828ddd53.
2. Castro FMP, Aquino R, Berti Júnior JA, Gonçalves LGC, Puggina EF. Strength training with vascular occlusion: a review of possible mechanisms. Hum Mov. 2017;18(2):3-14; doi: 10.1515/humo-2017-0010.

3. Kraemer WJ, Marchitelli L, Gordon SE, Harman E, Dziados JE, Mello R, et al. Hormonal and growth factor responses to heavy resistance exercise protocols. $\mathrm{J}$ Appl Physiol. 1990;69(4):1442-1450; doi: 10.1152/ jappl.1990.69.4.1442.

4. Ratamess NA, Falvo MJ, Mangine GT, Hoffman JR, Faigenbaum AD, Kang J. The effect of rest interval length on metabolic responses to the bench press exercise. Eur J Appl Physiol. 2007;100(1):1-17; doi: 10.1007/ s00421-007-0394-y.

5. Fink J, Schoenfeld BJ, Nakazato K. The role of hormones in muscle hypertrophy. Phys Sportsmed. 2018;46(1): 129-134; doi: 10.1080/00913847.2018.1406778.

6. Mohamad NI, Nosaka K, Cronin J. Maximizing hypertrophy: possible contribution of stretching in the interset rest period. Strength Cond J. 2011;33(1):81-87; doi: 10.1519/SSC.0b013e3181fe7164.

7. Souza AC, Bentes CM, de Salles BF, Reis VM, Alves JV, Miranda $\mathrm{H}$, et al. Influence of inter-set stretching on strength, flexibility and hormonal adaptations. J Hum Kinet.2013;36:127-135; doi:10.2478/hukin-2013-0013.

8. Rubini EC, Costa AL, Gomes PS. The effects of stretching on strength performance. Sports Med. 2007;37(3): 213-224; doi: 10.2165/00007256-200737030-00003.

9. Di Mauro HS, Moriggi R Junior, Dias SC, de Matos JM, Urtado CB. Ten seconds of passive stretching reduces the maximum strength. Man Ther Posturology Rehabil J. 2015;13(296):1-5; doi: 10.17784/mtprehabjournal.2015.13.296.

10. Do Rosário Souza AC, Borges Bastos CL, de Nazaré Dias Portal M, de Salles BF, Gomes TM, da Silva Novaes J. Acute effect of passive rest intervals and stretching exercise on multiple set performance [in Portuguese]. Rev Bras Cineantropom Desempenho Hum. 2009;11(4): 435-443.

11. Sforzo GA, Touey PR. Manipulating exercise order affects muscular performance during a resistance exercise training session. J Strength Cond Res. 1996;10(1): 20-24; doi: 10.1519/00124278-199602000-00004.

12. Avela J, Finni T, Liikavainio T, Niemelä E, Komi PV. Neural and mechanical responses of the triceps surae muscle group after $1 \mathrm{~h}$ of repeated fast passive stretches. J Appl Physiol. 2004;96(6):2325-2332; doi: 10.1152/ japplphysiol.01010.2003.

13. Cramer JT, Housh TJ, Coburn JW, Beck TW, Johnson GO. Acute effects of static stretching on maximal eccentric torque production in women. J Strength Cond Res. 2006;20(2):354-358; doi: 10.1519/R-18105.1.

14. Nelson AG, Kokkonen J, Arnall DA. Acute muscle stretching inhibits muscle strength endurance performance. J Strength Cond Res. 2005;19(2):338-343; doi: 10.1519/R-15894.1. 
15. Gomes TM, Simão R, Marques MC, Costa PB, da Silva Novaes J. Acute effects of two different stretching methods on local muscular endurance performance. J Strength Cond Res. 2011;25(3):745-752; doi: 10.1519/JSC.0b013e 3181cc236a.

16. Fowles JR, Sale DG, MacDougall JD. Reduced strength after passive stretch of the human plantarflexors. J Appl Physiol. 2000;89(3):1179-1188; doi: 10.1152/jappl.2000.89.3.1179.

17. Funk DC, Swank AM, Mikla BM, Fagan TA, Farr BK. Impact of prior exercise on hamstring flexibility: a comparison of proprioceptive neuromuscular facilitation and static stretching. J Strength Cond Res. 2003;17(3): 489-492; doi: 10.1519/1533-4287(2003)017<0489:IO $\mathrm{PEOH}>2.0 . \mathrm{CO} ; 2$.

18. Lopes CR, Soares EG, Santos ALR, Aoki MS, Marchetti PH. Effects of passive stretching protocol on multiple sets performance in resistance training [in Portuguese]. Rev Bras Med Esporte. 2015;21(3):224229; doi: 10.1590/1517-869220152103145780.

19. Ribeiro AS, Romanzini M, Dias DF, Ohara D, da Silva DR, Achour A Jr, et al. Static stretching and performance in multiple sets in the bench press exercise. J Strength Cond Res. 2014;28(4):1158-1163; doi: 10.1519/JSC.0000000000000257.

20. Ottaway CA, Husband AJ. The influence of neuroendocrine pathways on lymphocyte migration. Immunol Today. 1994;15(11):511-517; doi: 10.1016/0167-5699 (94)90206-2.

21. Brenner I, Shek PN, Zamecnik J, Shephard RJ. Stress hormones and the immunological responses to heat and exercise. Int J Sports Med. 1998;19(2):130-143; doi: 10.1055/s-2007-971895.

22. Kindig CA, Poole DC. Sarcomere length-induced alterations of capillary hemodynamics in rat spinotrapezius muscle: vasoactive vs passive control. Microvasc Res. 2001;61(1):64-74; doi: 10.1006/mvre.2000.2284.

23. Mayhew DL, Thyfault JP, Koch AJ. Rest-interval length affects leukocyte levels during heavy resistance exercise. J Strength Cond Res. 2005;19(1):16-22; doi: 10.1519/R-14113.1.

24. Kruse NT, Silette CR, Scheuermann BW. Influence of passive stretch on muscle blood flow, oxygenation and central cardiovascular responses in healthy young males. Am J Physiol Heart Circ Physiol. 2016;310(9):H1210H1221; doi: 10.1152/ajpheart.00732.2015.

25. Otsuki A, Fujita E, Ikegawa S, Kuno-Mizumura M. Muscle oxygenation and fascicle length during passive muscle stretching in ballet-trained subjects. Int J Sports Med.2011;32(7):496-502; doi:10.1055/s-0031-1275297.

26. McCully KK. The influence of passive stretch on muscle oxygen saturation. Adv Exp Med Biol. 2010;662:317322; doi: 10.1007/978-1-4419-1241-1_45.

27. Basualto-Alarcón C, Jorquera G, Altamirano F, Jaimovich $\mathrm{E}$, Estrada M. Testosterone signals through mTOR and androgen receptor to induce muscle hypertrophy. Med Sci Sports Exerc. 2013;45(9):1712-1720; doi: 10.1249/MSS.0b013e31828cf5f3.
28. Nalbandian M, Takeda M. Lactate as a signaling molecule that regulates exercise-induced adaptations. Biology. 2016;5(4):38; doi: 10.3390/biology5040038.

29. Oishi Y, Tsukamoto H, Yokokawa T, Hirotsu K, Shimazu M, Uchida K, et al. Mixed lactate and caffeine compound increases satellite cell activity and anabolic signals for muscle hypertrophy. J Appl Physiol. 2015;118(6): 742-749; doi: 10.1152/japplphysiol.00054.2014.

30. Walsh GS. Effect of static and dynamic muscle stretching as part of warm up procedures on knee joint proprioception and strength. Hum Mov Sci. 2017;55:189195; doi: 10.1016/j.humov.2017.08.014. 